\section{UCDNN}

LIBRARY
University of Connecticut OpenCommons@UConn

UCHC Articles - Research

University of Connecticut Health Center Research

$4-2012$

\title{
Bevacizumab and Breast Cancer: What Does the Future Hold?
}

Christina E. Stevenson

University of Connecticut School of Medicine and Dentistry

Follow this and additional works at: https://opencommons.uconn.edu/uchcres_articles

Part of the Medicine and Health Sciences Commons

\section{Recommended Citation}

Stevenson, Christina E., "Bevacizumab and Breast Cancer: What Does the Future Hold?" (2012). UCHC Articles - Research. 165. https://opencommons.uconn.edu/uchcres_articles/165 
Published in final edited form as:

Future Oncol. 2012 April ; 8(4): 403-414. doi:10.2217/fon.12.22.

\title{
Bevacizumab and breast cancer: what does the future hold?
}

\author{
Christina E Stevenson ${ }^{1}$, Masayuki Nagahashi ${ }^{2,3}$, Subramaniam Ramachandran ${ }^{2,3}$, Akimitsu \\ Yamada $^{2,3}$, Harry D Bear ${ }^{\star}, 2$, and Kazuaki Takabe ${ }^{2,3}$ \\ ${ }^{1}$ Department of Surgery, University of Connecticut Health Center, Farmington, CT 06030, USA \\ ${ }^{2}$ Division of Surgical Oncology, Department of Surgery, Virginia Commonwealth University School \\ of Medicine and the Massey Cancer Center, Richmond, VA 23298, USA
}

${ }^{3}$ Department of Biochemistry \& Molecular Biology, Virginia Commonwealth University School of Medicine \& the Massey Cancer Center, Richmond, VA 23298, USA

\begin{abstract}
Breast cancer is a major health concern for many women, but despite the current standard therapies, many women still die of metastatic disease. Angiogenesis has been evaluated as a possible target for therapy and bevacizumab (Avastin ${ }^{\circledR}$, Genentech/Roche, CA, USA), a monoclonal antibody against VEGF-A, has been developed to target this. Current clinical trials utilizing bevacizumab have shown an increase in progression-free survival, but this has not translated to an increase in overall survival in breast cancer patients. In this article, we summarize the currently published trials utilizing bevacizumab in the treatment of breast cancer and describe various methods of measuring angiogenesis in vitro and in vivo. We also describe the related process of lymphangiogenesis, as this may contribute to the mechanism of cancer progression and may be a potential target for therapy in the future. Understanding these processes may help us develop new treatments for breast cancer.
\end{abstract}

\section{Keywords}

angiogenesis; bevacizumab; breast cancer; clinical trial; lymphangiogenesis

Breast cancer is the second most common cancer occurring in women in the USA, with one in eight diagnosed with the disease during their lifetime [1]. Every year, approximately 230,000 new cases of invasive breast cancer will be diagnosed, and approximately 40,000 women will die of this disease [1]. Therefore, the search for new therapies to combat breast cancer is of great importance to women's health. Therapeutic interventions target various components that contribute to cancer progression and metastasis including angiogenesis, the generation of new blood vessels. Here, we will review the data from recently concluded clinical trials on angiogenesis-targeted therapy utilizing bevacizumab, a humanized monoclonal antibody that recognizes and blocks VEGF-A.

(C) 2012 Future Medicine Ltd

*Author for correspondence: Tel.: +1 804628 3242, Fax: +1 804828 4808, hdbear@vcu.edu.

Financial \& competing interests disclosure

The authors have no other relevant affifiliations or financial involvement with any organization or entity with a financial interest in or financial conflict with the subject matter or materials discussed in the manuscript apart from those disclosed.

No writing assistance was utilized in the production of this manuscript. 


\section{Role of angiogenesis in cancer progression}

Judah Folkman described the concept of tumor angiogenesis as a requirement for tumor growth in 1971 [2]. It has long been accepted that tumor growth beyond a diameter of a few millimeters requires generation of new vessels from existing ones. Folkman observed that this new growth of vessels in a tumor is even more vigorous than that observed in fresh wounds or inflammatory beds. He hypothesized that there is interplay between the tumor cells and the proliferating vascular endothelial cells, and that a solid tumor obtains its new blood vessels from the host. Folkman showed that a tumor implant of 2-3 $\mathrm{mm}$ in diameter, consisting of less than 1 million cells, induces DNA synthesis in the local, previously quiescent endothelial cells of capillaries and venules in as little as $6 \mathrm{~h}$ after tumor implantation [2]. He observed that this proliferation continued even when the tumor was separated from the vasculature by a filter that prevented the passage of cells. This result implied that the stimulus for this growth had to be a factor, or factors, secreted by the cells that diffuse across a tissue bed. This mechanism is also thought to contribute to the development of metastatic disease. Folkman demonstrated that factors released by tumors mediate angiogenesis [3], and he isolated a factor that he termed tumor angiogenesis factor, which caused endothelial cell proliferation and capillary formation, from various tumors [2]. He theorized that blocking the action of factors such as tumor angiogenesis factor could arrest tumor growth, and he coined the term 'antiangiogenesis' to describe the prevention of generation of new vessels in a tumor [2].

Vascular permeability factor, later termed VEGF, was first discovered in 1983 and was later shown to be secreted by tumors and to stimulate angiogenesis in a similar way to tumor angiogenesis factor [4]. VEGF has been identified as an ideal target for Folkman's proposed antiangiogenesis treatment, as this will theoretically inhibit growth of the primary tumor as well as metastatic tumors. Weidner et al. showed that a higher density of blood vessels in a tumor is an independent and significant prognostic indicator for overall and relapse-free survival in patients with early-stage breast cancer [5]. Multiple proangiogenic proteins that are produced by tumor cells have been identified, and VEGF is the major or sole proangiogenic protein expressed by breast cancers in $60 \%$ of women at the time of first diagnosis [6].

VEGF was later termed VEGF-A, with the discovery of multiple isoforms, and has been the most extensively studied [7]. It was found to play a significant role in the process of angiogenesis, and was discovered to be regulated by HIF1-a [7,8]. VEGF-A and its isoforms, VEGF-B, VEGF-C and VEGF-D, were found to act on two tyrosine kinase receptors, termed VEGF receptor-1 and VEGF receptor-2 [7]. The mechanisms of action of these other isoforms have not yet been studied in great detail, but are also thought to play a role in angiogenesis and lymphangiogenesis (Figure 1) [7,8].

It has been shown since 1991 that an anti-VEGF antibody reduces the density of the blood vessels in a given microscopic area in a tumor, termed the microvessel density, and causes inhibition of growth of some tumors in nude mice, which rely on VEGF as their sole mediator of angiogenesis [9]. This observation led to the recent development of bevacizumab, a humanized monoclonal antibody that binds VEGF-A, and thus is an indirect inhibitor of angiogenesis.

\section{Experimental assays to measure angiogenesis}

In order to develop and evaluate the potential benefit of new antiangiogenesis treatments, reliable methods of measuring angiogenesis both in vitro and in vivo are required. The ideal angiogenesis assay would be robust, reproducible, rapid and, most importantly, relate directly to results seen in the clinical setting. Most in vitro assays utilize isolated blood 
endothelial cells (BECs) and focus on their proliferation, migration and tube formation, all of which are essential for the process of angiogenesis. The isolation procedures of BECs from different organs have recently become increasingly sophisticated and organ-specific endothelial cells are now available as primary cell lines from commercial sources [10]. However, these cultures should be used with caution since BECs rapidly lose their physiological properties on repeated culturing [10].

Angiogenesis does not occur unless the BECs proliferate. A common method to measure cell proliferation is the 3-(4,5-dimethylthiazol-2-yl)-2,5-diphenyltetrazolium bromide or water-soluble tetrazolium salt assay (Roche, CA, USA) [11], which measure mitochondrial activity as a reflection of cell viability, or the $\left[{ }^{3} \mathrm{H}\right]$ thymidine incorporation assay that directly measures DNA synthesis and, hence, proliferation [12]. Critical parameters to consider are cell passage number and cell density. In addition to proliferation, BECs need to migrate into the perivascular stroma for angiogenesis to occur, which is commonly evaluated using a scratch assay or a Boyden chamber assay. The scratch assay, also known as a wound healing assay, consists of counting cells that migrate into the empty space produced by simple scraping of a confluent monolayer of BECs [13], thus it is considered an assessment of random cell mobility. The Boyden chamber assay utilizes a two-chambered apparatus separated with a filter, with cells placed on top and chemoattractants in the bottom, known as a Boyden chamber [14]. This assay assesses direct migration, or invasion, into filters coated with a basement membrane component such as collagen or Matrigel ${ }^{\mathrm{TM}}$ (BD Biosciences, NJ, USA) [15].

The most specific in vitro assay to measure angiogenesis is the tube formation assay, which measures the length of the capillary-like tubular structures that BECs form after being plated on basement membrane extracts such as Matrigel $[16,17]$. The key to the success of this assay is usage of a growth factor-reduced Matrigel, which avoids basal stimulation of BECs to form tubes [18], and ensuring BECs are in early passage (two to six passages) and approximately $80 \%$ confluent before harvesting them for use in the assay. Numerous reviews discuss tube formation assays in greater detail $[17,19,20]$. Recently, some researchers have utilized 3D assays, which are presumed to provide a more physiological model of the differentiation and tube formation of BECs [21,22].

Several ex vivo organ culture-based angiogenesis assays have been developed. The most described is the rat aortic ring assay, which monitors the vessels sprouting from thin rings of the rat aorta embedded in a collagen matrix [23]. This method can be used to monitor lymphangiogenesis when the thoracic duct is used in place of the aorta [24]. The most commonly used in vivo assay is the Matrigel plug assay, in which Matrigel is injected subcutaneously into mice in its liquid form at $4^{\circ} \mathrm{C}$, forming a gel at body temperature. Incorporation of various angiogenic factors in the gel matrix leads to the recruitment of BECs and formation of a fine tubular network, where angiogenesis can be evaluated with histological staining or injection of high-molecular weight dextran. A disadvantage of this technique is variability between different samples due to the inconsistencies in formation of the Matrigel plug despite injection of equal volumes.

This problem was solved in the directed in vivo angiogenesis assay where the Matrigel is enclosed in a silicone cylinder with one open end, termed an 'angioreactor' [25]. The angioreactor is filled with Matrigel, with or without test factors, and is implanted subcutaneously into mice. The number of BECs that migrate into it can be quantified with fluorescein isothiocyanate-dextran [25]. The directed in vivo angiogenesis assay is considered to be the most quantitative assay to measure angiogenesis in vivo. Other assays utilizing the chick chorioallantoic membrane [26] and corneal micropocket assay [27] provide an in vivo environment to test the effect on angiogenesis. These techniques are 
widely used, but they are labor intensive and the results can be inconsistent because of artifacts.

\section{Clinical trials with bevacizumab}

To date, bevacizumab has been tested in a number of trials of patients with metastatic, locally advanced or operable breast cancer. The published trials vary with respect to the dose and regimen of bevacizumab that is given, the agents it is combined with and the stage of disease being treated. In these studies, bevacizumab has been used as salvage or neoadjuvant therapy, as well as adjuvant treatment in operative cases.

\section{Early-phase studies}

One study has tested the addition of bevacizumab to endocrine therapy, since it is hypothesized that this may delay or prevent the development of resistance to endocrine therapy. Traina et al. treated patients in a Phase I open-label study with locally advanced or metastatic breast cancer treated with the aromatase inhibitor letrozole $2.5 \mathrm{mg}$ daily plus bevacizumab $15 \mathrm{mg} / \mathrm{kg}$ every 3 weeks. In all, 43 patients were treated with a median of 13 cycles. A total of $84 \%$ had at least stable disease, and four patients (9\%) had partial responses, while none had a complete response. Stable disease for longer than 24 weeks was noted in 29 patients (67\%) and the median progression-free survival (PFS) was 17.1 months. The overall clinical benefit rate was 77\% [28]. Ramaswamy et al. utilized bevacizumab with docetaxel as first- or second-line therapy for patients with metastatic breast cancer. A total of 27 patients received bevacizumab $10 \mathrm{mg} / \mathrm{kg}$ on days 1 and 15 with docetaxel $35 \mathrm{mg} / \mathrm{m}^{2}$ on days 1,8 and 15 of a 28 -day cycle. A median of six cycles were given per patient. Of these, $22 \%$ of the patients had failed prior chemotherapy. The overall response rate was $52 \%$, with median response duration of 6.0 months and median PFS of 7.5 months [29].

Multiple other studies have evaluated the addition of bevacizumab as salvage therapy in Phase II studies for patients who have failed prior chemotherapy. Dickler et al. treated 38 patients with metastatic breast cancer with erlotinib $150 \mathrm{mg}$ daily, and bevacizumab $15 \mathrm{mg} /$ kg every 3 weeks. The patients had received one or two prior chemotherapy regimens. One patient had a partial response for more than 52 months. A total of 15 patients had stable disease at first evaluation at 9 weeks and four of these patients sustained the response for more than 26 weeks. The median time to progression was 11 weeks. Overall, activity was limited [30]. Burstein et al. treated patients who had received one or two prior chemotherapy regimens for metastatic breast cancer or whose cancer had recurred within 12 months of adjuvant therapy and had measurable disease, with bevacizumab $10 \mathrm{mg} / \mathrm{kg}$ every 2 weeks and vinorelbine $25 \mathrm{mg} / \mathrm{m}^{2}$ every week. A total of 56 women were treated, with a $34 \%$ response rate and median time to progression of 5.5 months. Extent of prior chemotherapy did not appear to relate to outcomes [31].

Some Phase II studies have combined bevacizumab with metronomic chemotherapy regimens. In one study by Dellapasqua et al., patients with advanced breast cancer received capecitabine $500 \mathrm{mg}$ three-times per day and cyclophosphamide $50 \mathrm{mg}$ daily with bevacizumab $10 \mathrm{mg} / \mathrm{kg}$ every 2 weeks. A total of 46 patients were assessed, yielding one complete response (2\%), 21 partial responses (46\%), 19 patients with stable disease (41\%) and five with progressive disease $(11 \%)$. Eight patients had long-term stabilization, defined by stable disease for greater than or equal to 24 weeks, producing an overall clinical benefit of $68 \%$. The median time to progression was 42 weeks in this population [32]. Garcia-Saenz et al., have addressed this question as well [33]. Patients who were refractory to anthracycline and taxane therapy with metastatic breast cancer were given cyclophosphamide $50 \mathrm{mg}$ daily, methotrexate $1 \mathrm{mg} / \mathrm{kg}$ every 14 days and bevacizumab 10 $\mathrm{mg} / \mathrm{kg}$ every 14 days. Trastuzumab was given for HER2-overexpressing tumors. A total of 
22 patients were evaluable. After a median follow-up of 7.7 months, no patients had a complete response, $31.8 \%$ had a partial response and $31.8 \%$ had stable disease for a prolonged duration of greater than or equal to 24 weeks. The clinical benefit rate was $63.6 \%$ and median PFS was 7.5 months. The median overall survival was 13.6 months [33].

The North Central Cancer Treatment Group trial N0432 tested bevacizumab combined with docetaxel and capecitabine as first-line chemotherapy for patients with metastatic breast cancer. Patients received bevacizumab $15 \mathrm{mg} / \mathrm{kg}$ and docetaxel $75 \mathrm{mg} / \mathrm{m}^{2}$ on day 1 and capecitabine $825 \mathrm{mg} / \mathrm{m}^{2}$ twice per day on days $1-14$ every 21 days for a median of seven cycles. A total of 45 patients were evaluated in all. Two patients had complete responses and 20 patients had partial responses, yielding an overall response rate of $49 \%$. Nine patients had stable disease for more than 6 months, giving a clinical benefit rate of $69 \%$. The median response duration was 11.8 months, and median overall survival was 28.4 months. The median PFS was 11.1 months [34].

\section{Randomized trials for metastatic breast cancer}

A large randomized Phase III trial by Miller et al. randomly assigned patients with metastatic breast cancer who previously had received treatment with an anthracycline and a taxane to receive capecitabine $2500 \mathrm{mg} / \mathrm{m}^{2}$ twice daily on days $1-14$ every 3 weeks, either alone or with bevacizumab $15 \mathrm{mg} / \mathrm{kg}$ on day 1 [35]. In all, 462 patients were enrolled in the study. The addition of bevacizumab significantly increased the response rate from 9.1 to $19.8 \%$, but this did not translate to a longer PFS (4.86 vs 4.17 months). The overall survival was 15.1 versus 14.5 months, which was not significantly different [35].

The Eastern Cooperative Oncology Group (ECOG) trial, E2100, was a large Phase III trial testing the use of bevacizumab for the initial treatment of metastatic breast cancer. A total of 722 patients were enrolled who had metastatic breast cancer and had not received any prior cytotoxic chemotherapy; however, prior hormonal therapy or adjuvant cytotoxic therapy was allowed. HER2-positive patients were eligible only if they had received trastuzumab and constituted a minority of patients in this study. Patients were randomized to receive paclitaxel $90 \mathrm{mg} / \mathrm{m}^{2}$ on days 1,8 and 15 every 4 weeks, either alone or with bevacizumab 10 $\mathrm{mg} / \mathrm{kg}$ on days 1 and 15 . The combination showed a significant prolongation of PFS compared with paclitaxel alone, with a median of 11.8 versus 5.9 months. The objective response rate increased from 21.2 to $36.9 \%$, but the overall median survival was similar between the two groups, 26.7 versus 25.2 months [36].

Another Phase III trial that employed bevacizumab was the AVADO trial [37]. This study looked at bevacizumab $(7.5$ or $15 \mathrm{mg} / \mathrm{kg}$ ) given every 3 weeks versus placebo with docetaxel as first-line treatment of HER2-negative locally recurrent or metastatic breast cancer. It was shown that only the higher dose of bevacizumab resulted in a superior median PFS of 10.1 months, compared with 8.2 months for placebo in an unstratified analysis ( $\mathrm{p}=$ 0.006). Also, patients who received the higher dose of bevacizumab had a significant improvement in response rates compared with placebo (64 vs $46 \%$; $p=0.001$ ). Interestingly, there was no significant increase in the toxicity of docetaxel with the addition of bevacizumab. A subset analysis of patients $\Varangle 65$ years of age showed there was a similar increase in survival, although this was not statistically significant [38]. There was also no significant increase in adverse events in this population.

The RIBBON-1 trial was a randomized Phase III trial utilizing bevacizumab as first-line treatment of patients with HER2-negative, locally recurrent or metastatic breast cancer. Over 1200 patients were enrolled and median PFS was shown to increase in all treatment groups receiving bevacizumab, although there was no increase in overall survival in any group [39]. RIBBON-2 was a Phase III study that compared bevacizumab with standard chemotherapy 
versus standard chemotherapy alone in HER2-negative metastatic breast cancer. In total, 684 patients were enrolled and a statistically significant benefit in PFS was seen, with an increase from 5.1 to 7.2 months. Again, no statistically significant difference was shown in overall survival. The safety profile and overall response rates were similar to those seen in prior studies [40].

Recently, the early results of the AVEREL trial were presented. This was a randomized Phase III trial that combined bevacizumab with trastuzumab and docetaxel as first-line therapy for the treatment of HER2-positive locally recurrent or metastatic breast cancer. A total of 421 patients were treated in this study, and the addition of bevacizumab increased PFS from 13.9 to 16.8 months, which was found to be statistically significant when evaluated by an Independent Review Committee [41]. In addition, when patients were stratified as being high-VEGF producers versus low-VEGF producers, those who were high producers were found to have a prolonged median PFS of 13.6 months when treated with bevacizumab compared with only 8.5 months for those treated with trastuzumab and docetaxel alone. This study shows potentially promising results, and further evaluation is pending to determine biomarkers that may help identify which patients may benefit.

\section{Trials in the neoadjuvant setting}

A few trials have evaluated the use of bevacizumab as initial therapy for metastatic breast cancer or as part of a neoadjuvant treatment regimen in patients with locally advanced breast cancer. One study evaluated the effect of bevacizumab as neoadjuvant therapy for patients with inflammatory breast cancer. Wedam et al. performed a pilot study and treated 21 patients with previously untreated stage III or IV inflammatory and locally advanced breast cancer with bevacizumab $15 \mathrm{mg} / \mathrm{kg}$ on day 1 for one cycle followed by six cycles of bevacizumab combined with doxorubicin $50 \mathrm{mg} / \mathrm{m}^{2}$ and docetaxel $75 \mathrm{mg} / \mathrm{m}^{2}$ on day 1 every 3 weeks [42]. Patients underwent surgery at a minimum of 4 weeks after the seventh cycle. Radiation therapy was started after complete recovery from surgery. Eight additional cycles of bevacizumab were given postoperatively every 3 weeks. Hormonal therapy was given as indicated. As assessed by imaging, 14 patients had a partial response and no patients had a complete response, yielding an overall response rate of $67 \%$. Five patients had stable disease and two had progressive disease. The median PFS was 25.3 months and overall survival was $90.5 \%$ at 1 year and $80.0 \%$ at 2 years [42]. Tumor biopsies and dynamic contrast-enhanced MRI were obtained at baseline and after cycles one, four and seven. After receiving bevacizumab alone, dynamic contrast-enhanced MRI showed a significant decrease in blood flow. This decrease was greater than that observed after the administration of chemotherapy, although a decrease persisted. There was also a decrease in the presence of the phosphorylated VEGF receptor in tumor cells and increased apoptosis, which persisted after the administration of chemotherapy.

Greil et al. evaluated the use of bevacizumab combined with capecitabine and docetaxel as neoadjuvant therapy for breast cancer [43]. Patients had received no prior systemic therapy and had invasive HER2-negative breast cancer, that was T2-T4c with any nodal stage $(\mathrm{N})$ and showed no evidence of metastatic disease. Patients were treated with six 21-day cycles of bevacizumab $15 \mathrm{mg} / \mathrm{kg}$ on day 1 of cycles one to five, docetaxel $75 \mathrm{mg} / \mathrm{m}^{2}$ on day 1 of each cycle, and capecitabine $950 \mathrm{mg} / \mathrm{m}^{2}$ twice daily for 14 days of each cycle. Surgery was performed 2-4 weeks after the completion of neoadjuvant therapy, and was followed by radiation, chemotherapy and hormonal therapy as determined by institutional standards. A total of 18 patients were included, 15 of which (83\%) were able to undergo breastconserving surgery. An objective response, either complete or partial, or stable disease was observed in $72 \%$ of patients, while only $22 \%$ had a pathologic complete response, which 
was comparable with that previously seen after treatment with capecitabine and docetaxel alone [43].

One study looked specifically at patients with unfavorable prognostic features, such as estrogen and progesterone receptor staining of less than $10 \%$ of cancer cells, and T2-4c. All patients were treated with epirubicin $25 \mathrm{mg} / \mathrm{m}^{2}$ on days 1 and 2, cisplatin $60 \mathrm{mg} / \mathrm{m}^{2}$ on day 1 , and fluorouracil $200 \mathrm{mg} / \mathrm{m}^{2}$ on day 1 to 21 for four courses. Paclitaxel $90 \mathrm{mg} / \mathrm{m}^{2}$ was given on days 1,8 and 15 every 28 days for three courses. Bevacizumab $10 \mathrm{mg} / \mathrm{kg}$ was given every 14 days for six courses during paclitaxel treatment. Patients with inflammatory endocrine-responsive breast cancer received chemotherapy plus endocrine therapy with letrozole $2.5 \mathrm{mg}$ daily $\pm \mathrm{GnRH}$ analog with respect to menopausal status. A total of 30 patients were evaluated and $25(83 \%)$ had a partial response. One patient $(3 \%)$ had a complete response, giving an overall response rate of $87 \%$. Two patients $(7 \%)$ had stable disease and two patients (7\%) had progressive disease. All patients underwent surgery, and a pathologic complete response was seen in ten patients (33\%), all of whom received bevacizumab [44].

Baar et al. also examined bevacizumab in the neoadjuvant setting [45]. They randomized 49 patients with inoperable breast cancer to receive either two cycles of docetaxel alone $35 \mathrm{mg} /$ $\mathrm{m}^{2}$ weekly for 6 weeks, or the addition of bevacizumab $10 \mathrm{mg} / \mathrm{kg}$ every other week for a total of 16 weeks, followed by docetaxel. The overall clinical response rate was $78 \%$. Nineteen patients in each arm became operable and three patients in each arm had a clinical response but were inoperable. Three patients in the docetaxel arm and one patient in the bevacizumab arm had progressive disease. One patient receiving bevacizumab also experienced a treatment-related death during neoadjuvant therapy. There was no significant difference between the groups in either 60-month PFS (47.3\% in docetaxel arm vs $37.2 \%$ in bevacizumab arm) or overall survival (57.1\% in the docetaxel arm vs $57.6 \%$ in the bevacizumab arm) [45].

The National Surgical Adjuvant Breast and Bowel Project (NSABP) B-40 trial has accrued 1206 patients to evaluate the use of bevacizumab in the neoadjuvant setting [46]. In this trial, operable HER2-negative breast cancer patients were randomized to receive docetaxel with or without the addition of capecitabine or gemcitabine, followed by doxorubicin plus cyclophosphamide. In addition, half received bevacizumab with the first six doses of chemotherapy preoperatively. Patients then underwent surgery, followed by an additional ten doses of bevacizumab for those who received it preoperatively. Results from this trial were recently published and demonstrated that the addition of bevacizumab to neoadjuvant chemotherapy significantly increased the rate of pathological complete response in the breast from 28.2 to $34.5 \%(p=0.02)$ [46]. The effect of bevacizumab was greater in patients with hormone receptor-positive cancers than in the triple-negative subset. Results from a similar trial (GeparQuinto) from the German Breast Group, also recently published, were curiously different from the B-40 results [47]. This trial utilized an anthracycline and docetaxel-based chemotherapy regimen in 1948 patients with HER2-negative early-stage or locally advanced breast cancer. They also observed a significant increase in pathological complete response (in breast and nodes) with the addition of bevacizumab in the overall study population ( 14.9 vs $18.4 \% ; \mathrm{p}=0.04$ ), but in the German trial the difference was greater in the 663 patients with the triple negative cancers ( 27.9 vs $39.3 \% ; p=0.003)$ [47].

\section{Future issues}

Patients with metastatic breast cancer are faced with a poor prognosis and the search for therapies to improve this is ongoing. The results of the E2100 trial led to accelerated temporary approval by the US FDA of bevacizumab plus paclitaxel as first-line therapy in 
patients with metastatic breast cancer in 2008 [101]. Recently, this approval was withdrawn because it was felt that, after evaluating further trials, bevacizumab does not prolong overall survival or provide a benefit in slowing disease progression. The available data indicate that bevacizumab for the treatment of HER2-negative metastatic breast cancer does not clearly provide a survival benefit, since there is an increase in PFS, but not overall survival (Table 1) [48-50]. Early results from a few studies have shown improvement in pathologic response rates, but this has not yet been shown to translate into an improvement in overall survival. Additional data are needed from these early trials.

The ATHENA trial, which is a registry trial, treated patients with locally recurrent or metastatic breast cancer, and has shown with long-term follow-up that continuation of single-agent bevacizumab in patients without progression can be done with minimal toxicity and possibly long-term disease control [51]. Median overall survival was 30.0 months in these patients versus 18.4 months for those who discontinued bevacizumab during or before their chemotherapy regimen. Recently, they have shown that the risk profile in patients older than 70 years of age is no different to that of younger patients except for additional hypertension, although this risk was manageable [52]. Given the long-term safety data, extended administration of bevacizumab could potentially be a feasible method for treatment that may provide a benefit in overall survival, and needs to be investigated further. Certain populations of patients have not been completely addressed with the current trials. The Cancer and Leukemia Group B (CALGB) has sponsored three trials, CALGB 40502, 40503 and 40603, which are continuing, despite the FDA's determination to examine the utility of bevacizumab in patients with hormone receptor-positive breast cancer receiving endocrine therapy and those with triple-negative breast cancer. Results from these trials may help to determine whether there is still a role for bevacizumab in the treatment of breast cancer.

There are several factors that may have contributed to the failure to show an overall survival benefit of bevacizumab. First, most of the patient populations being studied are ones that have proven to be resistant to conventional therapies and thus may represent a more aggressive biology. Second, it is difficult to compare the results of these trials given the variety of treatment regimens utilized. Since there is no standardized method of administration of bevacizumab, many patients had limited administration due to toxicity, and the major end point for most is not overall survival. Finally, selection of patients for bevacizumab is challenging, because we lack reliable biomarkers to evaluate the activity for the agents. Studies have shown a relationship with HER2 overexpression and VEGF expression in human cancer cells in vitro [53]. A Phase I study looked at utilizing the combination of bevacizumab and trastuzumab to treat patients who are HER2-positive and showed no prohibitive toxicities with utilizing two humanized monoclonal antibodies, and there was a 55\% overall response rate identified in these patients, which is promising [54]. The identification of biomarkers such as HER2 that may identify patients who might benefit from bevacizumab may be the key to determining which patients should receive this therapy $[48,49]$. This might be accomplished with the use of tissues collected in neoadjuvant trials such as B-40 and GeparQuinto, although both of these studies excluded HER2-positive breast cancers.

In addition, the question must be addressed regarding whether PFS is a reliable end point to study. The majority of studies utilizing bevacizumab have only been able to show a prolongation in PFS, but this has not translated to overall survival. Other factors may be contributing to the lack of overall survival, such as the biology of the individual tumors being studied and the other therapies utilized in a treatment regimen aside from bevacizumab. Further study is needed to see if these differences are sustainable in certain populations. 
Finally, as is the case for any potent drug, bevacizumab is also not free from adverse effects. The most common side effects reported with bevacizumab include headache, nausea, hypertension and wound infection. Other more serious and sometimes fatal side effects can include left ventricular systolic dysfunction, gastrointestinal perforation, thromboembolism, hemorrhage or sepsis. The initial decision for approval of bevacizumab was mainly based on results from the E2100 trial, which reported similar adverse hematologic, gastrointestinal and musculoskeletal effects for both groups [36]. Side effects that were more common in the group that received bevacizumab were wound infection, fatigue, headaches and hypertension. There were no serious adverse events reported except for cerebrovascular ischemia in $1.9 \%$ of patients receiving bevacizumab, and so it was felt that the toxicities of bevacizumab was minimal and similar to other prior reports. Since the approval of bevacizumab, other trials have reported more significant side effects; based on the additional data, the FDA decided that its severe side effects outweighed the benefits of this drug. That, along with the lack of improvement in overall survival, led to the approval being withdrawn [102]. Of particular concern has been an increase in left ventricular dysfunction, as shown by a recently published meta-analysis [55].

\section{Emerging new target: lymphangiogenesis}

Although the critical impact of lymph node metastasis on prognosis and the progression of breast cancer through lymph node metastasis has been well established in patients [56], the active involvement of the lymphatic system in cancer metastasis has been revealed only recently, following the identification of molecular markers of lymphatic vessel development $[57,58]$. Together with the fact that lymphatic vessels are the main route of drainage of extracellular fluid, lymphangiogenesis, the generation of new lymphatic vessels from preexisting ones, has recently emerged as a new target for cancer therapy [57].

Lymphangiogenesis has been found to be controlled by VEGF-C and -D, which are associated with a number of human tumor types [58]. For metastasis to the draining lymph nodes to occur, the cancer cells need to access the draining lymphatic vessels to reach the regional lymph nodes, and tumor-induced lymphangiogenesis appears to promote this process by increasing the number of entry sites into the lymphatic system [59]. In fact, recent evidence suggests that lymphangiogenesis plays a key role in the development of nodal metastases from various human malignancies [60]. Tumor-induced lymphatic vessel density has correlated with metastasis to the draining lymph nodes, distant metastases and poor prognosis $[58,61-63]$. These studies suggest that expression of lymphangiogenic growth factors by tumor cells induces lymphangiogenesis, which promotes metastasis via lymphatic vessels to draining lymph nodes and beyond.

VEGF-C also promotes circumferential enlargement of the collecting vessels, leading to increased lymph flow and transport of tumor cells, as well as accommodation of larger tumor cell clusters [64-66]. Experimental inhibition of this process in animal models has suggested that lymphangiogenic growth factors facilitate the metastatic spread of tumor cells via lymphatic vessels [65]. Furthermore, lymphangiogenic growth factors produced by the primary tumor appear to act at a distance by inducing lymphangiogenesis, or sinusoidal hyperplasia, in the sentinel lymph node before the arrival of the first metastatic cells $[67,68]$. Expansion of the lymphatic vasculature in premetastatic lymph nodes has been confirmed in mouse models of breast carcinomas [69], indicating that lymphangiogenesis in the premetastatic lymph node fosters tumor dissemination by creating a favorable environment for cancer cells.

As described in previous sections, several clinical trials have demonstrated that not all patients respond to bevacizumab, and those that do can acquire resistance [70]. Furthermore, 
patients with high levels of VEGF-C expression were shown to have worse outcomes [71]. Based upon the fact that VEGF-C plays a key role in lymphangiogenesis, it is possible that a contributing factor in the poor outcomes of some patients may be due to a failure to inhibit the effects of VEGF on lymphangiogenesis [72].

Given the fact that draining lymph nodes are usually the first site of metastasis, targeting lymphangiogenesis and nodal metastasis is expected to be a key new target for the development of new therapies for cancer. Considering the strong associations among tumorinduced lymphangiogenesis, regional lymphatic spread, distant metastasis and survival, the inhibition of tumor lymphatic vessels seems to be an attractive target. Inhibition of lymphangiogenic factors or receptors has had a significant antimetastatic efficacy in several experimental models (Figure 1) [66,73]. Moreover, a combination of chemotherapy and antilymphangiogenic therapy might be more effective in eliminating metastatic cells while preventing the rebuilding of the damaged lymphatic vasculature [74]. Ramucirumab, a novel compound that targets both angiogenesis and lymphangiogenesis, is currently being evaluated in a Phase I clinical trial and is reported to have an impressive, albeit early, effect on both stable disease and partial responses in a variety of tumor types [75]. Moreover, the first inhibitor of lymphangiogenesis, the VEGF receptor-3-targeting monoclonal antibody IMC-3C5, has recently entered a Phase I clinical trial for patients with advanced solid tumors [76,103]. In the future, tailored treatments consisting of combinations of chemotherapy and targeted therapy against both angiogenesis and lymphangiogenesis are expected to improve patient outcomes. This progression to the clinic may be guided by new avenues of research such as the identification of biomarkers that predict response to treatment.

\section{Conclusion}

Bevacizumab is an angiogenesis inhibitor that has shown promising results in the treatment of various cancers. Its role in the treatment of breast cancer is not clearly defined by the current trials; despite improvement in PFS, this has not translated into improvement in overall survival. Further studies are needed to address whether longer-term administration of bevacizumab will show significant improvement in overall survival for patients with metastatic breast cancer. The development of methods of evaluation of angiogenesis will allow us to monitor the effectiveness of such therapies. Also, the identification of biomarkers may delineate which patients will benefit from antiangiogenic therapy. These therapies, and new ones in development, may also function to inhibit lymphangiogenesis and, thereby, inhibit the progression and development of metastatic disease.

\section{Future perspective}

Agents for the treatment of breast cancer are continuing to evolve. Currently, the role for antiangiogenic agents in the treatment of breast cancer is not well defined. In the future we may be able to develop biomarkers that may help us better identify which patients will benefit from certain treatments and we will therefore be able to provide individualized therapy for patients. Targeting a combination of the mechanisms of cancer growth and metastasis, including lymphangiogenesis, will be an exciting frontier for the development of new agents in the treatment of breast cancer.

\section{Acknowledgments}

This article has been funded by a Virginia Commonwealth University Grant (BIRCWH K12HD055881) and a Susan G Komen for the Cure Career Catalyst Research Grant (KG090510) to K Takabe. 


\section{References}

Papers of special note have been highlighted as:

- of interest

- of considerable interest

1. Siegel R, Ward E, Brawley O, Jemal A. Cancer statistics, 2011: the impact of eliminating socioeconomic and racial disparities on premature cancer deaths. CA Cancer J. Clin. 2011; 61(4): 212-236. [PubMed: 21685461]

2. Folkman J. Tumor angiogenesis: therapeutic implications. N. Engl. J. Med. 1971; 285(21):11821186. [PubMed: 4938153]

3. Folkman J. Anti-angiogenesis: new concept for therapy of solid tumors. Ann. Surg. 1972; 175(3): 409-416. [PubMed: 5077799]

4. Dvorak HF, Brown LF, Detmar M, Dvorak AM. Vascular permeability factor/vascular endothelial growth factor, microvascular hyperpermeability, and angiogenesis. Am. J. Pathol. 1995; 146(5): 1029-1039. [PubMed: 7538264]

5. Weidner N, Folkman J, Pozza F, et al. Tumor angiogenesis: a new significant and independent prognostic indicator in early-stage breast carcinoma. J. Natl Cancer Inst. 1992; 84(24):1875-1887. [PubMed: 1281237]

6. Folkman J. Fundamental concepts of the angiogenic process. Curr. Mol. Med. 2003; 3(7):643-651. [PubMed: 14601638]

7. Ferrara N, Hillan KJ, Novotny W. Bevacizumab (Avastin), a humanized anti-VEGF monoclonal antibody for cancer therapy. Biochem. Biophys. Res. Commun. 2005; 333(2):328-335. [PubMed: 15961063]

8. Ho QT, Kuo CJ. Vascular endothelial growth factor: biology and therapeutic applications. Int. J. Biochem. Cell Biol. 2007; 39(7-8):1349-1357. [PubMed: 17537667]

9. Folkman J. Angiogenesis and breast cancer. J. Clin. Oncol. 1994; 12(3):441-443. [PubMed: 7509850]

10. Staton CA, Reed MW, Brown NJ. A critical analysis of current in vitro and in vivo angiogenesis assays. Int. J. Exp. Pathol. 2009; 90(3):195-221. [PubMed: 19563606]

11. Denizot F, Lang R. Rapid colorimetric assay for cell growth and survival. Modifications to the tetrazolium dye procedure giving improved sensitivity and reliability. J. Immunol. Methods. 1986; 89(2):271-277. [PubMed: 3486233]

12. Yu CH, Wu J, Su YF, et al. Anti-proliferation effect of 3-amino-2-imino-3,4-dihydro-2 H-1,3benzothiazin-4-one (BJ-601) on human vascular endothelial cells: G0/G1 p21-associated cell cycle arrest. Biochem. Pharmacol. 2004; 67(10):1907-1916. [PubMed: 15130767]

13. Wong MK, Gotlieb AI. In vitro reendothelialization of a single-cell wound. Role of microfilament bundles in rapid lamellipodia-mediated wound closure. Lab. Invest. 1984; 51(1):75-81. [PubMed: 6376946]

14. Boyden $\mathrm{S}$. The chemotactic effect of mixtures of antibody and antigen on polymorphonuclear leucocytes. J. Exp. Med. 1962; 115:453-466. [PubMed: 13872176]

15. Albini A, Benelli R, Noonan DM, Brigati C. The 'chemoinvasion assay': a tool to study tumor and endothelial cell invasion of basement membranes. Int. J. Dev. Biol. 2004; 48(5-6):563-571. [PubMed: 15349831]

16. Kubota Y, Kleinman HK, Martin GR, Lawley TJ. Role of laminin and basement membrane in the morphological differentiation of human endothelial cells into capillary-like structures. J. Cell Biol. 1988; 107(4):1589-1598. [PubMed: 3049626]

17. Arnaoutova I, George J, Kleinman HK, Benton G. The endothelial cell tube formation assay on basement membrane turns 20: state of the science and the art. Angiogenesis. 2009; 12(3):267-274. [PubMed: 19399631]

18. Kleinman HK, Martin GR. Matrigel: basement membrane matrix with biological activity. Semin. Cancer Biol. 2005; 15(5):378-386. [PubMed: 15975825] 
19. Arnaoutova I, Kleinman HK. In vitro angiogenesis: endothelial cell tube formation on gelled basement membrane extract. Nat. Protoc. 2010; 5(4):628-635. [PubMed: 20224563]

20. Ucuzian AA, Greisler HP. In vitro models of angiogenesis. World J. Surg. 2007; 31(4):654-663. [PubMed: 17372665]

21. Koh W, Stratman AN, Sacharidou A, Davis GE. In vitro three dimensional collagen matrix models of endothelial lumen formation during vasculogenesis and angiogenesis. Methods Enzymol. 2008; 443:83-101. [PubMed: 18772012]

22. Benton G, George J, Kleinman HK, Arnaoutova IP. Advancing science and technology via 3D culture on basement membrane matrix. J. Cell. Physiol. 2009; 221(1):18-25. [PubMed: 19492404]

23. Aplin AC, Fogel E, Zorzi P, Nicosia RF. The aortic ring model of angiogenesis. Methods Enzymol. 2008; 443:119-136. [PubMed: 18772014]

24. Bruyere F, Melen-Lamalle L, Blacher S, et al. Modeling lymphangiogenesis in a three-dimensional culture system. Nat. Methods. 2008; 5(5):431-437. [PubMed: 18425139]

25. Guedez L, Rivera AM, Salloum R, et al. Quantitative assessment of angiogenic responses by the directed in vivo angiogenesis assay. Am. J. Pathol. 2003; 162(5):1431-1439. [PubMed: 12707026]

26. Miller WJ, Kayton ML, Patton A, et al. A novel technique for quantifying changes in vascular density, endothelial cell proliferation and protein expression in response to modulators of angiogenesis using the chick chorioallantoic membrane (CAM) assay. J. Transl. Med. 2004; 2(1): 4. [PubMed: 14754458]

27. Rogers MS, Birsner AE, D’Amato RJ. The mouse cornea micropocket angiogenesis assay. Nat. Protoc. 2007; 2(10):2545-2550. [PubMed: 17947997]

28. Traina TA, Rugo HS, Caravelli JF, et al. Feasibility trial of letrozole in combination with bevacizumab in patients with metastatic breast cancer. J. Clin. Oncol. 2009; 28(4):628-633. [PubMed: 19841327]

29. Ramaswamy B, Elias AD, Kelbick NT, et al. Phase II trial of bevacizumab in combination with weekly docetaxel in metastatic breast cancer patients. Clin. Cancer Res. 2006; 12(10):3124-3129. [PubMed: 16707611]

30. Dickler MN, Rugo HS, Eberle CA, et al. A Phase II trial of erlotinib in combination with bevacizumab in patients with metastatic breast cancer. Clin. Cancer Res. 2008; 14(23):7878-7883. [PubMed: 19047117]

31. Burstein HJ, Chen YH, Parker LM, et al. VEGF as a marker for outcome among advanced breast cancer patients receiving anti-VEGF therapy with bevacizumab and vinorelbine chemotherapy. Clin. Cancer Res. 2008; 14(23):7871-7877. [PubMed: 19047116]

32. Dellapasqua S, Bertolini F, Bagnardi V, et al. Metronomic cyclophosphamide and capecitabine combined with bevacizumab in advanced breast cancer. J. Clin. Oncol. 2008; 26(30):4899-4905. [PubMed: 18794539]

33. Garcia-Saenz JA, Martin M, Calles A, et al. Bevacizumab in combination with metronomic chemotherapy in patients with anthracycline- and taxane-refractory breast cancer. J. Chemother. 2008; 20(5):632-639. [PubMed: 19028628]

34. Perez EA, Hillman DW, Dentchev T, et al. North Central Cancer Treatment Group (NCCTG) N0432: Phase II trial of docetaxel with capecitabine and bevacizumab as first-line chemotherapy for patients with metastatic breast cancer. Ann. Oncol. 2009; 21(2):269-274. [PubMed: 19901014]

35. Miller KD, Chap LI, Holmes FA, et al. Randomized Phase III trial of capecitabine compared with bevacizumab plus capecitabine in patients with previously treated metastatic breast cancer. J. Clin. Oncol. 2005; 23(4):792-799. [PubMed: 15681523] • One of the first large randomized trials to show that the addition of bevacizumab resulted in a significant increase in response rate.

36. Miller K, Wang M, Gralow J, et al. Paclitaxel plus bevacizumab versus paclitaxel alone for metastatic breast cancer. N. Engl. J. Med. 2007; 357(26):2666-2676. [PubMed: 18160686] Results from the Eastern Cooperative Oncology Group trial E2100, a Phase III trial that tested the use of bevacizumab in addition to paclitaxel for initial treatment of metastatic breast cancer, which increased progression-free survival and the rates of response, but not overall survival.

37. Miles DW, Chan A, Dirix LY, et al. Phase III study of bevacizumab plus docetaxel compared with placebo plus docetaxel for the first-line treatment of human epidermal growth factor receptor 2negative metastatic breast cancer. J. Clin. Oncol. 2010; 28(20):3239-3247. [PubMed: 20498403] • 
Reports the results from the AVADO trial which showed that high-dose bevacizumab increased progression-free survival and response rate without an increase in toxicity.

38. Pivot X, Schneeweiss A, Verma S, et al. Efficacy and safety of bevacizumab in combination with docetaxel for the first-line treatment of elderly patients with locally recurrent or metastatic breast cancer: results from AVADO. Eur. J. Cancer. 2011; 12:12.

39. Robert NJ, Dieras V, Glaspy J, et al. RIBBON-1: randomized, double-blind, placebo-controlled, Phase III trial of chemotherapy with or without bevacizumab for first-line treatment of human epidermal growth factor receptor 2-negative, locally recurrent or metastatic breast cancer. J. Clin. Oncol. 2011; 29(10):1252-1260. [PubMed: 21383283]

40. Brufsky AM, Hurvitz S, Perez E, et al. RIBBON-2: a randomized, double-blind, placebocontrolled, Phase III trial evaluating the efficacy and safety of bevacizumab in combination with chemotherapy for second-line treatment of human epidermal growth factor receptor 2-negative metastatic breast cancer. J. Clin. Oncol. 2011; 29(32):4286-4293. [PubMed: 21990397]

41. Gianni L, Romieu G, Lichinitser M, et al. First results of AVEREL, a randomized Phase III trial to evaluate bevacizumab (BEV) in combination with trastuzumab $(\mathrm{H})+\operatorname{docetaxel}(\mathrm{DOC})$ as first-line therapy for HER2-positive locally recurrent/metastatic breast cancer (LR/mBC). Cancer Res. 2011 (Abstract S4-8).

42. Wedam SB, Low JA, Yang SX, et al. Antiangiogenic and antitumor effects of bevacizumab in patients with inflammatory and locally advanced breast cancer. J. Clin. Oncol. 2006; 24(5):769777. [PubMed: 16391297]

43. Greil R, Moik M, Reitsamer R, et al. Neoadjuvant bevacizumab, docetaxel and capecitabine combination therapy for HER2/neu-negative invasive breast cancer: efficacy and safety in a Phase II pilot study. Eur. J. Surg. Oncol. 2009; 35(10):1048-1054. [PubMed: 19250795]

44. Balduzzi A, Montagna E, Bagnardi V, et al. Infusional fluorouracil, epirubicin, and cisplatin followed by weekly paclitaxel plus bevacizumab in locally advanced breast cancer with unfavorable prognostic features. Anticancer Drugs. 2009; 20(3):197-203. [PubMed: 19182679]

45. Baar J, Silverman P, Lyons J, et al. A vasculature-targeting regimen of preoperative docetaxel with or without bevacizumab for locally advanced breast cancer: impact on angiogenic biomarkers. Clin. Cancer Res. 2009; 15(10):3583-3590. [PubMed: 19417018]

46. Bear HD, Tang G, Rastogi P, et al. Bevacizumab added to neoadjuvant chemotherapy for breast cancer. N. Engl. J. Med. 2012; 366:310-320. [PubMed: 22276821] - Results of National Surgical Adjuvant Breast and Bowel Project B-40 that demonstrating that the addition of bevacizumab to neoadjuvant chemotherapy significantly increases the pathological complete response rate.

47. von Minckwitz G, Eidtmann H, Rezai M, et al. Neoadjuvant chemotherapy and bevacizumab for HER2-negative breast cancer. N. Engl. J. Med. 2012; 366(4):299-309. [PubMed: 22276820] Results from the GeparQuinto clinical trial also showing increased pathologic complete response rates with the addition of bevacizumab to neoadjuvant chemotherapy.

48. Yang SX. Bevacizumab and breast cancer: current therapeutic progress and future perspectives. Expert Rev. Anticancer Ther. 2009; 9(12):1715-1725. [PubMed: 19954282]

49. Keefe SM, Demichele A. The expanding role of bevacizumab in the treatment of human epidermal growth factor receptor 2-negative breast cancer. Curr. Oncol. Rep. 2010; 12(1):22-25. [PubMed: 20425604]

50. Koutras AK, Fountzilas G, Makatsoris T, Peroukides S, Kalofonos HP. Bevacizumab in the treatment of breast cancer. Cancer Treat. Rev. 2010; 36(1):75-82. [PubMed: 19932567]

51. Smith I, Pierga JY, Biganzoli L, et al. Final overall survival results and effect of prolonged ( $>/=1$ year) first-line bevacizumab-containing therapy for metastatic breast cancer in the ATHENA trial. Breast Cancer Res.Treat. 2011; 130(1):133-143. [PubMed: 21830015]

52. Biganzoli L, Di Vincenzo E, Jiang Z, et al. First-line bevacizumab-containing therapy for breast cancer: results in patients aged $>=70$ years treated in the ATHENA study. Ann. Oncol. 2012; 23(1):111-118. [PubMed: 21444356]

53. Epstein M, Ayala R, Tchekmedyian N, Borgstrom P, Pegram MD, Slamon D. HER2overexpressing human breast cancer xenografts exhibit increased angiogenic potential modiated by vascular endothelial growth factor (VEGF). Breast Cancer Res. Treat. 2002; 76:S143-S143. 
54. Pegram M, Yeon C, Ku N, Gaudreault J, Slamon D. Phase I combined biological therapy of breast cancer using two humanized monoclonal antibodies directed against HER2 proto-oncogene and vasucular endothelial growth factor (VEGF). Breast Cancer Res. Treat. 2004; 88:S124-S125.

55. Choueiri TK, Mayer EL, Je Y, et al. Congestive heart failure risk in patients with breast cancer treated with bevacizumab. J. Clin. Oncol. 2011; 29(6):632-638. [PubMed: 21205755] — First meta-analysis to show that bevacizumab is associated with an increased risk of significant heart failure.

56. Edge, SB.; Byrd, DR.; Compton, CC., et al., editors. AJCC Cancer Staging Manual (7th Edition). NY, USA: Springer; 2010.

57. Nagahashi M, Ramachandran S, Rashid OM, Takabe K. Lymphangiogenesis: a new player in cancer progression. World J. Gastroenterol. 2010; 16(32):4003-4012. [PubMed: 20731013]

58. Tammela T, Alitalo K. Lymphangiogenesis: molecular mechanisms and future promise. Cell.

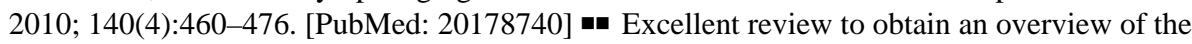
field of lymphangiogenesis, written by one of the world leaders in this field.

59. Mumprecht V, Detmar M. Lymphangiogenesis and cancer metastasis. J. Cell. Mol. Med. 2009; 13(8A):1405-1416. [PubMed: 19583813]

60. Nagahashi M, Shirai Y, Wakai T, Sakata J, Ajioka Y, Hatakeyama K. Perimuscular connective tissue contains more and larger lymphatic vessels than the shallower layers in human gallbladders. World J. Gastroenterol. 2007; 13(33):4480-4483. [PubMed: 17724804]

61. Rinderknecht M, Detmar M. Tumor lymphangiogenesis and melanoma metastasis. J. Cell Physiol. 2008; 216(2):347-354. [PubMed: 18481261]

62. Van der Auwera I, Cao Y, Tille JC, et al. First international consensus on the methodology of lymphangiogenesis quantification in solid human tumours. Br. J. Cancer. 2006; 95(12):16111625. [PubMed: 17117184]

63. Pepper MS, Tille JC, Nisato R, Skobe M. Lymphangiogenesis and tumor metastasis. Cell Tissue Res. 2003; 314(1):167-177. [PubMed: 12883995]

64. Alitalo K, Tammela T, Petrova TV. Lymphangiogenesis in development and human disease. Nature. 2005; 438(7070):946-953. [PubMed: 16355212]

65. He Y, Rajantie I, Pajusola K, et al. Vascular endothelial cell growth factor receptor 3-mediated activation of lymphatic endothelium is crucial for tumor cell entry and spread via lymphatic vessels. Cancer Res. 2005; 65(11):4739-4746. [PubMed: 15930292]

66. Hoshida T, Isaka N, Hagendoorn J, et al. Imaging steps of lymphatic metastasis reveals that vascular endothelial growth factor-C increases metastasis by increasing delivery of cancer cells to lymph nodes: therapeutic implications. Cancer Res. 2006; 66(16):8065-8075. [PubMed: 16912183]

67. Hirakawa S, Brown LF, Kodama S, Paavonen K, Alitalo K, Detmar M. VEGF-C-induced lymphangiogenesis in sentinel lymph nodes promotes tumor metastasis to distant sites. Blood. 2007; 109(3):1010-1017. [PubMed: 17032920]

68. Hirakawa S, Kodama S, Kunstfeld R, Kajiya K, Brown LF, Detmar M. VEGF-A induces tumor and sentinel lymph node lymphangiogenesis and promotes lymphatic metastasis. J. Exp. Med. 2005; 201(7):1089-1099. [PubMed: 15809353]

69. Qian CN, Berghuis B, Tsarfaty G, et al. Preparing the 'soil': the primary tumor induces vasculature reorganization in the sentinel lymph node before the arrival of metastatic cancer cells. Cancer Res. 2006; 66(21):10365-10376. [PubMed: 17062557]

70. Jain RK, Duda DG, Clark JW, Loeffler JS. Lessons from Phase III clinical trials on anti-VEGF therapy for cancer. Nat. Clin. Pract. Oncol. 2006; 3(1):24-40. [PubMed: 16407877]

71. Jubb AM, Miller KD, Rugo HS, et al. Impact of exploratory biomarkers on the treatment effect of bevacizumab in metastatic breast cancer. Clin. Cancer Res. 2011; 17(2):372-381. [PubMed: 21224365]

72. Tvorogov D, Anisimov A, Zheng W, et al. Effective suppression of vascular network formation by combination of antibodies blocking VEGFR ligand binding and receptor dimerization. Cancer Cell. 2010; 18(6):630-640. [PubMed: 21130043] 
73. Roberts N, Kloos B, Cassella M, et al. Inhibition of VEGFR-3 activation with the antagonistic antibody more potently suppresses lymph node and distant metastases than inactivation of VEGFR-2. Cancer Res. 2006; 66(5):2650-2657. [PubMed: 16510584]

74. Ran S, Volk L, Hall K, Flister MJ. Lymphangiogenesis and lymphatic metastasis in breast cancer. Pathophysiology. 2010; 17(4):229-251. [PubMed: 20036110]

75. Spratlin JL, Mulder KE, Mackey JR. Ramucirumab (IMC-1121B): a novel attack on angiogenesis.

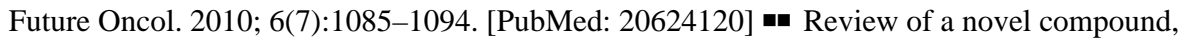
ramucirumab, which targets both angiogenesis and lymphangiogenesis.

76. Alitalo K. The lymphatic vasculature in disease. Nat. Med. 2011; 17(11):1371-1380. [PubMed: 22064427]

\section{Websites}

101. NCI. FDA Approval for Bevacizumab. 2008. www.cancer.gov/cancertopics/druginfo/fdabevacizumab\#Anchor-Metastati-43353

102. US FDA. Proposal to withdraw approval for the breast cancer indication for Avastin (bevacizumab). 2011. www.fda.gov/downloads/NewsEvents/Newsroom/UCM280546.pdf

103. A study of anti-VEGFR-3 monoclonal antibody IMC-3C5 in subjects with advanced solid tumors. 2012. http://clinicaltrials.gov/ct2/show/NCT01288989 


\section{Executive summary}

\section{Role of angiogenesis in cancer progression}

- VEGF-A is a major proangiogenic protein expressed in a majority of breast cancers.

- An antibody to VEGF-A has been shown to decrease microvessel density convincingly in preclinical models.

\section{Experimental assays to measure angiogenesis}

- Several methods of measuring cell proliferation, migration and invasion have been developed for use in vivo and in vitro, but can be very labor intensive and inconsistent.

\section{Clinical trials with bevacizumab}

- Multiple randomized Phase III trials have shown that the addition of bevacizumab to various chemotherapy regimens improves overall response and progression-free survival in most of the trials for the treatment of breast cancer, but no study has shown a significant improvement in overall survival.

- Reports of toxicity have ranged from mild side effects of headache, hypertension or nausea, to fatal side effects of gastrointestinal perforation or hemorrhage.

- The National Surgical Adjuvant Breast and Bowel Project B-40 and GeparQuinto trials have shown that bevacizumab added to chemotherapy may increase pathologic complete response rates in certain patient populations.

- Current ongoing trials are being performed to study certain patient populations (e.g., hormone receptor-positive, HER2-negative or HER2positive) to define a potential role for bevacizumab.

\section{Emerging new target: lymphangiogenesis}

- Lymphangiogenesis plays a key role in the development of lymph node metastases and is regulated in part by VEGF-C.

- Antilymphangiogenic agents may be required to prevent progression of disease more effectively in patients with breast cancer. 


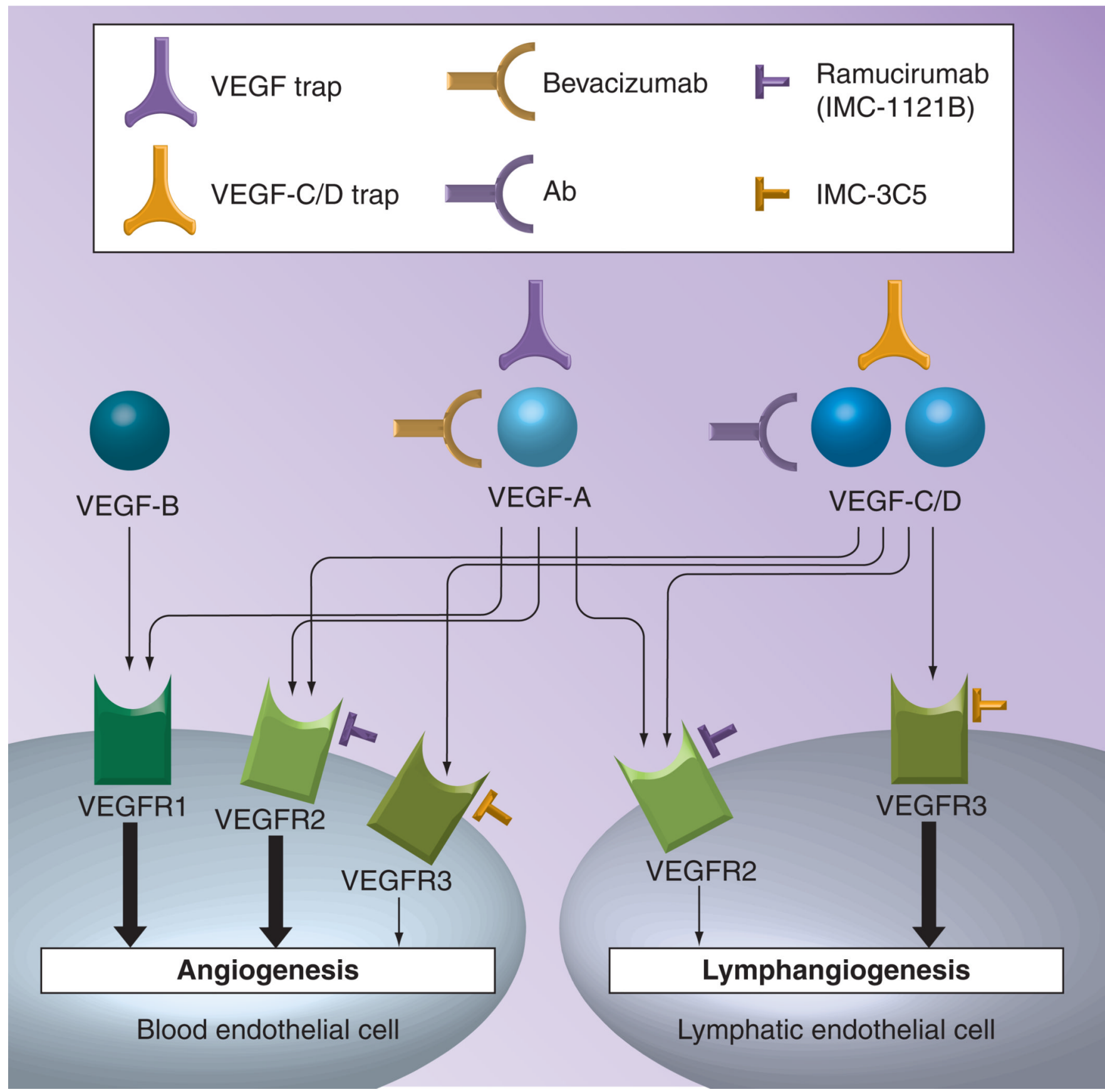

Figure 1. Inhibitors of VEGF-A, -B, -C/D, and their receptors

Bevacizumab is a monoclonal antibody against VEGF-A. VEGF and VEGF-C/D traps are fusion proteins that bind to VEGF-A, or VEGF-C/D, respectively. Ramucirumab (IMC-1121B) and IMFC-3C5 are monoclonal antibodies against VEGF receptor-2 and VEGF receptor-3, respectively. Thick arrows express major pathways and thin arrows express reported, but minor, pathways.

Ab: Antibody; VEGFR: VEGF receptor. 


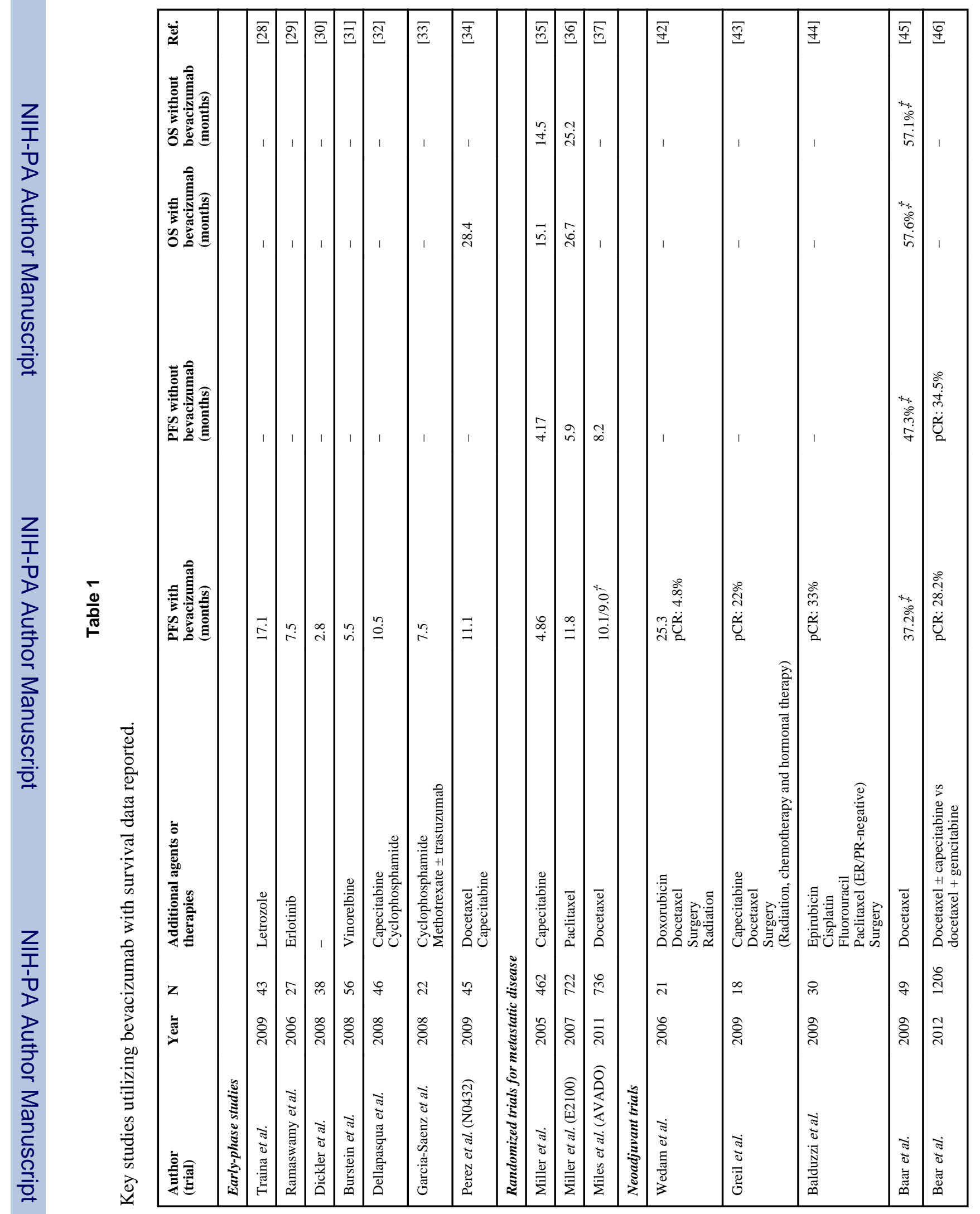

Future Oncol. Author manuscript; available in PMC 2013 February 01. 


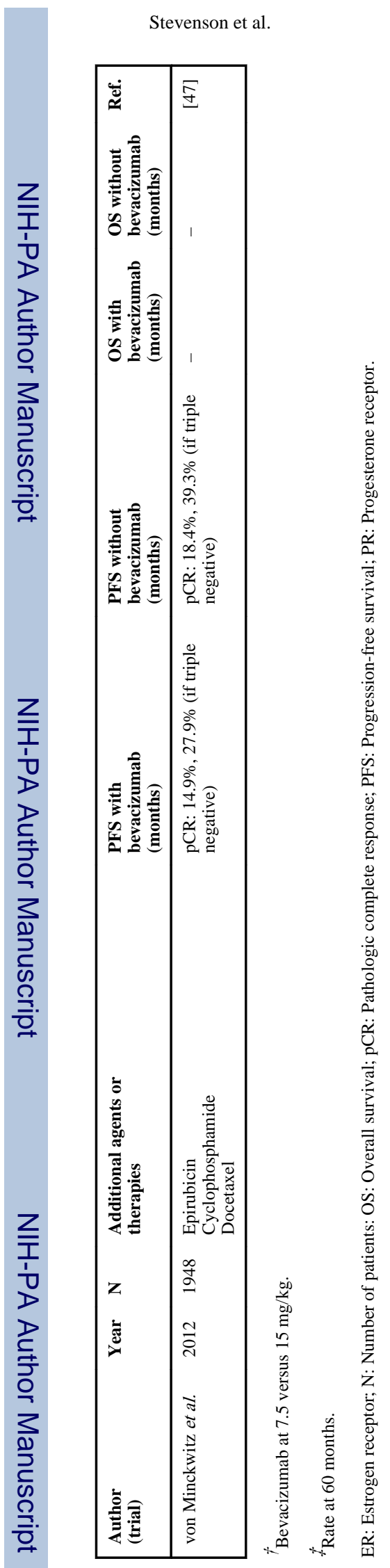

Page 19

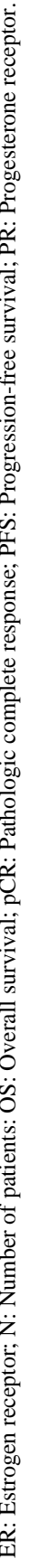

Future Oncol. Author manuscript; available in PMC 2013 February 01. 